\title{
Entropy of the Retina Template
}

\author{
A. Arakala ${ }^{1}$, J.S. Culpepper ${ }^{1}$, J. Jeffers ${ }^{1}$, A. Turpin ${ }^{1}$, S. Boztaş ${ }^{1}$, \\ K.J. Horadam ${ }^{1}$, and A.M. McKendrick ${ }^{2}$ \\ 1 RMIT University, Melbourne, Australia \\ 2 University of Melbourne, Parkville, Australia \\ \{arathi.arakala, shane.culpepper, jason. jeffers, andrew.turpin, \\ serdar.boztas, kathy.horadam\}@rmit.edu.au, allisonm@unimelb.edu.au
}

\begin{abstract}
We compare two vessel extraction methods for creation of a retina template, using a database of 20 images of normal retinas. Each vessel in a well defined region is represented by a three dimensional feature, from which a retina template is built. Based on the sample distributions, we propose a preliminary theoretical model to predict the entropy of a retina template. We analyse by experimental and theoretical means the entropy present, and infer that entropy from our retina template compares sufficiently favourably with that of a minutia-based fingerprint template to warrant further study.
\end{abstract}

\section{Introduction}

Over the past two decades there has been increasing research and community interest in biometric identification, with a growing installed base of biometric devices and a general community acceptance of biometric based authentication. As research in the area has grown, so has the breadth of the biometric characteristics considered for authentication purposes. Interestingly, despite retina-based authentication being one of the early techniques to be explored and successfully commercialized, the volume of published research in the field has been relatively low, though research interest in it has increased noticeably in the last few years. The lack of a large standard database of retinal images for evaluating matching algorithms has meant that it is difficult to compare the performance of different algorithms, or to understand properly the error performance an algorithm in isolation, or even to decide whether further investment in the area is justified. It is expected that matching algorithms will improve significantly over time. In this paper we use an alternative approach to evaluate the information content captured by one of the key algorithms of interest in the field and compare the result to mature fingerprint and iris based techniques.

Section 2 provides background on retina-based authentication and in Section 3 we compare two medical retina image-processing techiques to determine a preferred feature extraction method. In Section 4 we adopt a slight modification of the construction of Farzin, Abrishami-Moghaddam, and Moin [4] to form the retina template. We describe the experiments conducted to study retinal entropy and the theoretical model of the template distribution we develop, giving our results in Section 5 . 


\section{Background}

The retina has many desirable characteristics as the basis of authentication. Retinal blood vessel patterns are known to be very distinctive [12, even between identical twins [14]. The blood vessel structure is very stable over time, well shielded from outside environmental impacts, and believed to be difficult to spoof [6]. From a privacy perspective, a retinal image is unlikely to be captured from an individual without their knowledge, unlike many leading biometrics such as face, fingerprint, voice, and even iris.

Retina-based authentication is perceived as intrusive and user-unfriendly, ever since the first commercially successful retina scanner (Hill [7/6]) required subjects to place their eye very close to the reader and focus on an alignment target as a focussed beam of light was shone in their eye. This process was also the reason retina-based authentication traditionally was not appropriate for individuals with poor eyesight. Now, however, the technological limitations which required an intrusive process no longer exist.

Retinal images appear to contain abundant identifying information and four broad approaches have emerged to capture this information: using general signal and image processing techniques on the raw retina image; matching of the branching blood vessel structure as a whole; matching crossing and bifurcation points of the blood vessel structure; and matching the pattern of vessels traversing a well defined region.

We will concentrate on the last approach but briefly discuss the first three. Tabataee, Fard and Jafariani 13 employ a retinal identification system based on features extracted from the raw image using an analytic Fourier-Mellin transform and image moments. In [9], Marino et al. propose a matching algorithm that focusses on the overall network structure of the blood vessel pattern. The authors store the entire image as the template and perform a cross correlation between the query image and the stored image, but the images are processed in such a way that it is the blood vessel network that is actually being correlated. A template based on the vessel network would be expected to be a natural evolution of this approach. Several research groups have used the points where blood vessels cross one another or bifurcate as the basis of a feature set to match individuals [15]16]10. This is analogous to successful fingerprint matching techniques.

\subsection{Matching Vessels Traversing a Region}

The original retina scanner [8] may be classified as using this type of approach. The author used a focussed beam to scan around the fovea, in effect measuring the the blood vessels that traversed a narrow annulus centered on the fovea.

More recently Farzin et al [4 used image processing to locate the optical disk and then extract the blood vessels that traverse an annulus centered on the optical disk, sort them into three different width ranges and match them to an enrolled template by measuring their angular position and angle relative to the border of the annulus. The authors test their algorithm on a database with 60 images, but rotate images randomly to provide more samples for matching. They 
do not state whether these rotated images are used to simulate multiple samples from the same subject, as would be obtained in a true enrolment situation.

\section{Retinal Image Processing}

Our database consists of single images of 20 clinically normal right eyes. The images typically show uneven illumination and poor contrast of minor vessels. These images were obtained from the reflectance images of a Scanning Laser Topographer (HRT, Heidelberg Engineering) which uses a low power laser to scan $384 \times 384$ pixel images at 32 different depths of the optic nerve head.

Following the approach of Farzin et al. 4, we extracted vessels that traversed an annulus described by two circles; the inner of radius 80 pixels and an outer of radius 130 pixels from the centre of the image. The center was located as the centroid of the lowest 150 pixels as given by the topographic map that accompanied each image. In order to extract the vessels we compared two methods. The first, due to Can et al. 215], uses aligned edge detection filters to locate parallel lines and label them as vessel boundaries. An example appears in Figure 1(a). The second, due to Sofka and Stewart [11, uses multi-scale matched filtering and assigns each pixel a "vesselness" score, and then uses training data to produce a likelihood ratio of the pixel being part of the vessel. An example appears in Figure 1(b). Both methods are implemented in publicly available software at www.cs.rpi.edu/ sofka/vessels_exec.html. Once the vessels have been labelled in the images, we extracted the annulus crossings by hand. If a vessel splits while traversing the annulus, we follow only the most anticlockwise vessel. This is an arbitrary choice, but as long as the choice is consistent it should not affect a matching process based on this extraction. After this stage, each vessel has four $(x, y)$ co-ordinates, one for each of the four corners of its crossing of the inner and outer circles. The final step is to "flatten" these vessel segments into two dimensions using polar co-ordinates, as shown in Figure 2. On average, the number of vessel segments extracted from images by Method 1 was 13, while

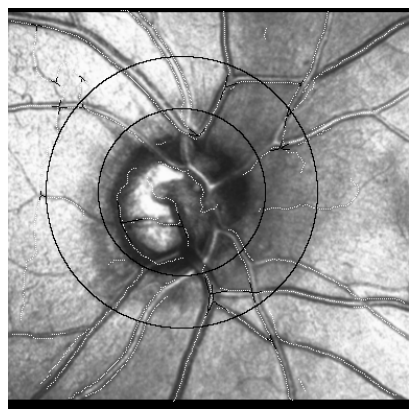

(a) Method 1

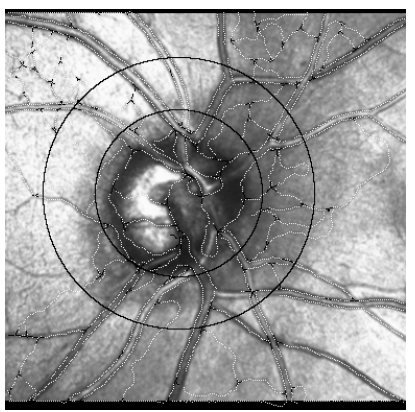

(b) Method 2

Fig. 1. Image and labelling typical of those used in this study 


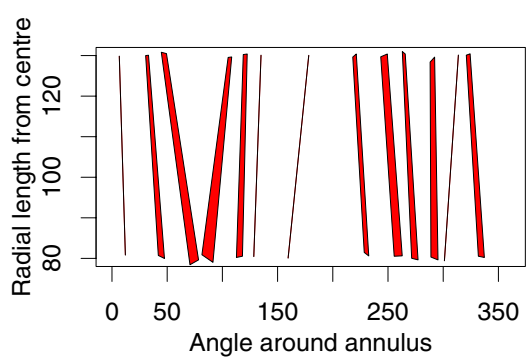

Fig. 2. Flattened extracted features typical of those used in this study

that for Method 2 was 18. We conclude that Method 2 has superior performance in vessel extraction from the annulus centred on the optic disc, confirming the approach in [11. Consequently we used the vessel segments extracted by Method 2 to build our biometric templates.

\section{Entropy of a Retina Template}

The entropy contained in a biometric template is a measure of the randomness or distinctiveness between the biometric templates of different people. The template itself can have a very different (usually lower) entropy when compared to the original biometric image, because template creation uses only a selection of the significant biometric image features that distinguish one person from another. The more significant features that are used in building a template, the greater will be the template entropy.

Entropy can be determined in two ways. One method is to fit the distribution of the features to existing theoretical models. The other is to determine empirically the probability $p$ that two templates from different people will match, by testing over a very large database of biometric samples. The entropy (in bits) of the biometric template is then $-\log _{2} p$.

Owing to the small size of our database, we adopt the first method to estimate the entropy of a retina template.

\subsection{Entropy of Fingerprint and Iris Templates}

The entropy in a fingerprint has been analysed by Zhu, Dass and Jain [17, who proposed a family of mixture models to simulate the occurrence of minutiae locations and orientations in a fingerprint. They simulated imposter matches and determined an equation for the probability $p$ that fingerprints from different people would match on certain numbers of minutiae 17. Their model was a close representation of their empirical results (from the FVC2000 DB1) when the match threshold was greater than 7 minutiae. Arakala, Horadam and Boztas [1] applied a simple bounding box technique to quantise the measurements of minutiae location and orientation with respect to the core for fingerprints obtained 
from the FVC2002 DB2 and found that the average number of quantised elements (resulting from well separated minutiae) in a fingerprint template was 38 . Using Zhu et al's results gives $p=9.3 \times 10^{-4}$ for templates having a comparable number of minutiae $(\approx 36)$ when the match theshold is 12 minutiae. This corresponds to approximately 10 bits of entropy, and approximates the maximum information that can be contained in a purely minutiae-based template.

On similar lines, Daugman 3] compared 1 billion irises from different people and at the most relaxed relative Hamming Distance threshold of 0.317 between the digital iris templates, found that the probability of obtaining a false match was roughly 0.000001 , corresponding to an entropy of 20 bits. These tests show that the specific iris template tested (IrisCode) has extremely high distinctiveness across populations. This allows it to be used to design iris based authentication systems with negligible false match rates.

\subsection{The Retina Template and Feature Distribution}

After the image processing steps described in Section 3, the vessel segments crossing the annulus are used to create the retina template as an ordered set of three-dimensional vectors. The $i^{\text {th }}$ feature (vessel segment) is represented by the triplet $\left(p_{i}, t_{i}, c_{i}\right)$, where $p_{i}$ is the position (in degrees around the annulus) of the segment, $t_{i}$ is its thickness in degrees and $c_{i}$ is its crossing angle (the angle in degrees the segment makes with a radial line drawn from the annulus, passing through the centroid of the segment).

These characteristics are the ones selected in [4. Further research is needed to determine if other characteristics, such as the number of vessel segments, are also important. In our sample, this number had mean 18.45 and standard deviation $\approx 3.245$, but the sample is too small to suggest a model distribution.

We analyse the templates obtained from our database to determine the parameters of the retinal entropy model developed in Section 4.3. For this model, it is necessary to assume that the retinal images are roughly pre-aligned. The results of Section 5.2 show this assumption may be too strong for current alignment and registration technology.

First we determine the sample distribution of each of the three components of a retinal feature independently. The frequency of occurrence of every possible value of position, thickness and crossing angle across every feature in every template of the database up to a resolution of $1^{\circ}$ was noted. The frequency distributions are given in Figure 3 .

\subsection{The Retinal Entropy Model}

We develop a theoretical model of the biometric templates informed by the empirical data. The key simplifying assumption we make is that the position, thickness, and crossing angle are all statistically independent of one another. This assumption appears reasonable given the data available. There are particular circumstances where this assumption is expected to break down. For example, we expect the thickest blood vessels to show a distinctive position distribution. 


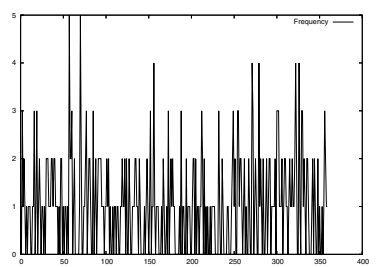

(a) Position

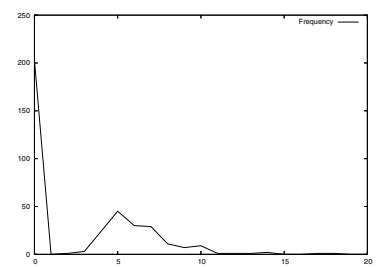

(b) Thickness

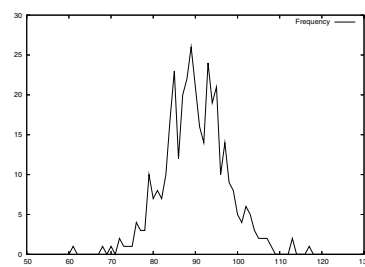

(c) Crossing Angle

Fig. 3. Frequency distributions of features

Figure 3(a), (b) and (c) show our observed distribution of position, thickness and crossing angle respectively. We chose to model the position as a uniform distribution and the crossing angle as a normal distribution. The crossing angles had a mean of $90^{\circ}$ and a standard deviation of $7.5^{\circ}$. For the thickness we chose to combine a spiked distribution for the thin vessels containing $55 \%$ of the vessels, and a normal distribution with a mean of 6.4 and a standard deviation of 2.4 for the remaining $45 \%$ of medium to thick vessels. The overall distribution of features is then modelled as the product of the three distributions.

Templates from the same retina will vary due to movement of the head during retina image capture and variation in the number of features extracted from each capture. To compensate, tolerances have to be specified in each dimension of a feature vector. Each dimension is separately quantised. The resulting number of quanta controls the tradeoff between accuracy and leniency in a matching process. The vector of resolution levels for the position, thickness and crossing angle that generate the quantised space is the quantisation triplet $\left(p_{t o l}, t_{t o l}, c_{t o l}\right)$. It is important for any matching system to determine the smallest permissible tolerances that minimise intra-sample variation and still retain distinctiveness between different retinas.

The model product distribution described above is used to generate a large number of synthetic templates, randomly. This allows us to match a much larger population of templates than can be obtained from current sample databases. Note that the number of features per synthetic template varies according to the model product distribution rather than the sample distribution. The synthetic templates are matched, and their match characteristics provide the entropy predicted by the model. The general procedure is as follows:

- A quantization triplet $\left(p_{t o l}, t_{t o l}, c_{t o l}\right)$ is chosen.

- A match threshold $s$ is chosen, where two templates are regarded as a match if they share at least $s$ quanta containing a feature.

- A large number of pairs of templates $N$ are generated and the number of matches $m$ is recorded.

Provided $N$ is large enough to result in actual matches, the entropy as predicted by the model is then

$$
\log _{2} N-\log _{2} m .
$$


For most of our tests $N$ is of the order of 100,000,000 and the match number $m$ is recorded for all values of $s$.

\section{Results and Analysis}

\subsection{Results for Comparison with [4]}

We first chose a quantization that matched the algorithm described by the authors in 4 as closely as possible, and was expected to result in an acceptable false non-match rate (FNMR). For position we chose $p_{t o l}=1^{\circ}$ exactly as in [4]. Fortunately, most of the entropy results from variation in position and consequently this is the most critical choice. For vessel thickness we chose 4 quanta of width 5 pixels, where one of the quanta is too far down the tail of the normal distribution to contribute meaningfully. The remaining three effectively partition the vessels into thin, medium and thick as in 4. Crossing angle is less directly comparable because in [4] the authors use a cosine-based correlation that cannot be directly related to our model. We chose 4 quanta of width $25^{\circ}$ based around the $90^{\circ}$ mean as the closest match to their correlation, given the slow decay of the cosine compared to the normally distributed crossing angle.

The results of running the model on this quantization choice over the range of values of $s$ is shown in Figure 4 as the line labelled by quantisation triplet $(1,5,25)$. In 4 the authors find acceptable FNMR up to a threshold of 9 as measured in their matching algorithm. Due to the correlation matching and weighting they employ there is no direct relationship with our model. As the cosine-based correlation decays slowly and thick vessels are weighted by a factor of 2 we should expect that this is equivalent in our model to an $s$ threshold of 4.5. At these threshold values our model predicts an entropy value in the range of at least 17.0 bits for this type of template. Such an entropy value would compare very favorably with fingerprints and quite well with iris (see Section 4.1).

As noted above, the choice of quantization of position is critical to ultimate entropy predicted by the model and the $1^{\circ}$ accuracy used in [4] is particularly strict. Having worked with our data, we doubt that acceptable FNMR would result from so strict a choice. The authors of [4] stated that extra images were generated by rotating existing images and this may explain why they could use such a strict choice without harming the FNMR. It is unlikely this would be replicated on a more representative database.

\subsection{Intra Sample Variation}

An estimate of intra sample variation is required to determine the most suitable quantisation triplet. As our original database contained only one image per individual, we obtained 5 image samples of a different retina, extracted the features using Method 2 and created their templates. For various quantisation triplets the number of quantised features that matched between two samples and the average number of features that matched across the $\left(\begin{array}{l}5 \\ 2\end{array}\right)=10$ different intra-sample comparisons were noted. Table 1 lists some of these quantisation triplets and the 
corresponding average number of matched features between templates. Observe that the quantisation triplet $(10,10,10)$ gives the highest average number, about half, of feature matches between samples of the same retina.

\subsection{Results and Analysis}

The probabilities of finding a feature in each quantum was computed from our database. In this small dataset, only $3-5 \%$ of quanta had non-zero probabilities and so were unable to use this distribution in the model directly. Consequently, we input each of the quantisation triplets in Table 1 into the theoretical model and used the assumed probability distributions in Section 4.3 to compute the entropy of a retina template at different match thresholds $s$. The results are given in Figure 4. They show a marked improvement in entropy at finer quantisation triplets. The feature distribution of position in Figure 3(a) confirms that position has a close to uniform distribution and it contributes significantly to retina template entropy. A finer quantisation in position will greatly enhance the maximum retina template entropy possible, but a practical limitation to this is the degree to which alignment and registration accuracy can be achieved. Farzin et al. 4] have performed their matching tests at $1^{\circ}$ resolution for position at a match threshold of $6-7$ features. In Table 1 (quantisation triplet $(1,5,25)$ ) we show that such a fine quantisation on position may only allow an average of 2 features in common between samples of the same retina.

However, if alignment and registration technology improves sufficiently that position can be aligned to $1^{\circ}$ accuracy, Figure 4 indicates that we should achieve over 20 bits of entropy, which will then be comparable to that of an iris template.

The third column in Table 1 shows the retina template entropies from Figure 4. for different quantisation triplets sampled at a threshold $s$ corresponding to the average number of intra sample feature matches. Choosing the average number of matches as the threshold will imply a FNMR of close to 50\%. This might overestimate the FNMR as the average number of matches has been obtained by conducting experiments on multiple samples of one retina only.

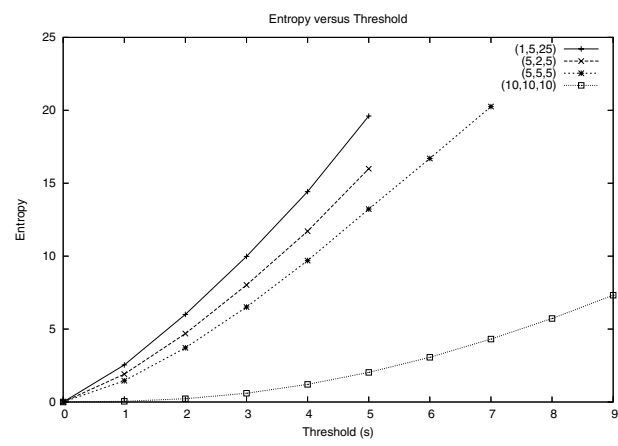

Fig. 4. Entropy (vertical) vs threshold (horizontal) for varying quantisation triplets 
Table 1. Quantisation Triplets, Intra Sample Match Average and Predicted Entropies

\begin{tabular}{|c|c|c|}
\hline $\begin{array}{c}\text { Quantisation Triplet } \\
\left(p_{\text {tol }}, t_{t o l}, c_{t o l}\right)\end{array}$ & $\begin{array}{c}\text { Average Number } \\
\text { of Intra Sample Matches }\end{array}$ & $\begin{array}{c}\text { Predicted } \\
\text { Entropy }\end{array}$ \\
\hline$(10,10,10)$ & 9 & 7.3 \\
$(5,5,5)$ & 6 & 16.7 \\
$(5,2,5)$ & 4 & 11.7 \\
$(1,5,25)$ & 2 & 6.0 \\
\hline
\end{tabular}

The quantisation triplet $(5,5,5)$ gives the highest entropy of 16.7 bits at the match threshold of $s=6$. This is significantly higher than that of fingerprint minutiae-based templates and close to that of iris templates.

\subsection{Conclusions and Future Work}

We have proposed a preliminary theoretical model, with three degrees of freedom, to compute the entropy of retina templates. Choosing quantisation triplets from empirical studies, we determined that retina templates having entropies greater than 16 bits can be created. This compares favourably with minutiaebased fingerprint templates but the model must be tested against much larger retina databases before general conclusions can be drawn. We have identified that position is the principle contributor to retina template entropy. This implies that the maximum possible retina template entropy is limited only by the accuracy of the registration and alignment of intra-retina samples and the limitations introduced by image processing.

Currently we are exploring the use of larger databases to obtain an empirical joint probability distribution to tune our theoretical model and compare with these results. We expect tests on larger databases to exhibit higher entropy, more comparable to that in an iris template. We may incorporate other characteristics, such as number of features, into the model. We also intend to construct retina templates using the three other methods of Section 2 and evaluate their template entropies using a similar modelling technique.

Acknowledgement. This research was supported by an RMIT College of SET Seeding Grant.

\section{References}

1. Arakala, A., Horadam, K.J., Boztas, S.: Practical Considerations for Secure Minutiae Based Templates. In: Proc. 2008 Biometrics Symposium, Tampa, Florida, September 23-25, pp. 53-58. IEEE Press, Los Alamitos (2008)

2. Can, A., Shen, H., Turner, J.N., Tanenbaum, H.L., Roysam, B.: Rapid automated tracing and feature extraction from live high-resolution retinal fundus images using direct exploratory algorithms. IEEE Trans. Inform. Technol. Biomed. 3(2), 125-138 (1999) 
3. Daugman, J.: Probing the Uniqueness and Randomness of IrisCodes: Results From 200 Billion Iris Pair Comparisons. Proc. IEEE 94(11), 1927-1935 (2006)

4. Farzin, H., Abrishami-Moghaddam, H., Moin, M.: A Novel Retinal Identification System. EURASIP Journal on Advances in Signal Processing, Article ID 280635, 10 (2008), doi:10.1155/2008/280635

5. Fritzsche, K., Can, A., Shen, H., Tsai, C., Turner, J., Tanenbuam, H., Stewart, C., Roysam, B.: Automated model based segmentation, tracing and analysis of retinal vasculature from digital fundus images. In: Suri, J.S., Laxminarayan, S. (eds.) State-of-The-Art Angiography, Applications and Plaque Imaging Using MR, CT, Ultrasound and X-rays, pp. 225-298. Academic Press, London (2003)

6. Hill, R.: Retinal Identification. In: Jain, A.K., Bolle, R.M. (eds.) Biometrics: Personal Identification in Networked Society, pp. 123-142. Kluwer Academic Press, Boston (1999)

7. Hill, R.: Apparatus and method for identifying individuals through their retinal vasculature patterns, US Patent No. 4109237 (1978)

8. Hill, R.: Rotating beam ocular identification apparatus and method, US Patent No. 4393366 (1983)

9. Marino, C., Penedo, M., Carreira, M., Gonzalez, F.: Retinal angiography based authentication. In: Sanfeliu, A., Ruiz-Shulcloper, J. (eds.) CIARP 2003. LNCS, vol. 2905, pp. 306-313. Springer, Heidelberg (2003)

10. Ortega, M., Penedo, M., Marino, C., Carreira, M.: Similarity metrics analysis for feature point based retinal authentication. In: Campilho, A., Kamel, M.S. (eds.) ICIAR 2008. LNCS, vol. 5112, pp. 1023-1032. Springer, Heidelberg (2008)

11. Sofka, M., Stewart, C.V.: Retinal Vessel Centerline Extraction Using Multiscale Matched Filters, Confidence and Edge Measures. IEEE Transactions on Medical Imaging 25(12), 1531-1546 (2006)

12. Simon, C., Goldstein, I.: A new scientific method of identification. New York State Journal of Medicine 35(18), 901-906 (1935)

13. Tabataee, H., Fard, A., Jafariani, H.: A novel human identifier system using retina image and fuzzy clustering approach. In: Proceedings of the 2nd IEEE International Conference on Information and Communications Technologies (ICTTA 2006), Damascus, Syria, pp. 1031-1036 (April 2006)

14. Tower, P.: The fundus oculi in monozygotic twins: report of six pairs of identical twins. Archives of Ophthalmology 54, 225-239 (1955)

15. Xu, Z., Guo, X., Hu, X., Cheng, X.: The blood vessel recognition of ocular fundus. In: Yeung, D.S., Liu, Z.-Q., Wang, X.-Z., Yan, H. (eds.) ICMLC 2005. LNCS, vol. 3930, pp. 4493-4498. Springer, Heidelberg (2006)

16. Xu, Z., Guo, X., Hu, X., Chen, X., Wang, Z.: The identification and recognition based on point for blood vessel of ocular fundus. In: Zhang, D., Jain, A.K. (eds.) ICB 2006. LNCS, vol. 3832, pp. 770-776. Springer, Heidelberg (2006)

17. Zhu, Y., Dass, S.C., Jain, A.K.: Statistical models for assessing the individuality of fingerprints. IEEE Transactions on Information Forensics and Security 2(3), 391-401 (2007) 